\title{
Case Report: Rare Presentations of Accidental Subdural Block in Labor Epidural Anesthesia
}

\author{
Jing Song, Atit Shah, Sujatha Ramachandran \\ Department of Anesthesiology, Montefiore Medical Center, Albert Einstein College of Medicine, Bronx, USA. \\ Email: jsong@montefiore.org
}

Received July $5^{\text {th }}$, 2012; revised August $8^{\text {th }}$, 2012; accepted August $27^{\text {th }}, 2012$

\begin{abstract}
The incidence of accidental injection or catheterization of the subdural space during performance of a neuroaxial block has recently increased. It can occur even when an experienced practitioner performs the neuraxial procedure. The presentation of numerous unexplainable clinical signs in the process of continuous epidural anesthesia, which do not fit the clinical picture of subarachnoid or intravascular injection, should envoke a high suspicion for unintentional subdural block. We report two cases of patients who achieved prolonged labor analgesia via epidural technique with only half the initial loading dose of local anesthetic. Both patients also had short episodes of hypotension. Additionally, one patient presented with severe hypoxemia and mild motor block of both upper and lower extremities. The other patient presented with transit unresponsiveness without motor block. Both patients rapidly responded to vasopressors. Desaturation in one patient, however, was persistent lasting for more than four hours. Her bedside chest X-ray was inconclusive "possible pulmonary edema" and the follow up Chest CT Scan on the second day revealed aspiration pneumonia. Based on the clinical findings, these two cases were suggestive of subdural block with cranial nerve involvement.
\end{abstract}

Keywords: Epidural Anesthesia; Subdural Block; Gestation; Aspiration

\section{Introduction}

Subdural space, a potential space between the arachnoid mater and dura mater, usually remains closed [1]. The incidence of subdural blockade during neuroaxial block is reported to be approximately of $0.82 \%$. Several recent studies of clinical findings analyzed with radiographic evaluation indicate that the incidence may be much higher than reported, ranging from $1 \%$ to $13 \%[2,3]$. The diagnosis of subdural blocks is difficult to make based on the clinical picture because of its varied presentation. Recently, algorithms have been developed which (Lubenow et al.'s diagnostic paradigm, Hoftman and Ferrante's four step algorithm, and an electrical stimulation of the epidural catheter application) provide strong strategies to facilitate diagnosis [2,4-6]. We present two cases of atypical demonslyration of subdural block during neuroaxial block for labor analgesia.

\subsection{Case 1}

A 32-year-old multigravida female who was 67 inches tall and weighed 183 pounds was admitted in her active labor at the 40th week of gestation. Her past medical history included cardiomegaly, anemia, and a urinary tract infection. She was allergic to Levoquin. At the time the labor epidural was requested, her blood pressure was 107/69 mmHg with pulse between 110 to 115 beats/min.

Following intravenous hydration of Ringer's lactate, the patient was seated; her L4-5 epidural space was easily identified with the loss-of-resistance to air technique using an 18-gauge Touhy needle. The bevel was oriented in the cephalad direction. An epidural catheter (multiport, open tip) was placed through the needle with minimal resistance. Aspiration of the catheter revealed no blood or cerebrospinal fluid. A test dose of $3 \mathrm{ml}$ of $1.5 \%$ Lidocaine and 1:200,000 epinephrine was injected and the epidural catheter was secured. There were no signs of any sensory or motor block and no significant vital sign change within 5 minutes after the test dose. A loading dose of $0.125 \%$ of Bupivicaine $10 \mathrm{ml}$ was administrated by slow incremental injection over 6 minutes after the patient returned to the supine position. Thirty minutes after the loading dose was given and before starting continual administration of local anesthetics, the patient described "throat itching" and dysphagia associated with a non-productive cough. Her systolic blood pressure dropped from 110 to $89 \mathrm{mmHg}$ and heart rate dropped from 110 to 100 beats/min. The possibility of an allergic reaction to local anesthetics was considered and the patient was immediately treated with aggressive hydration, $12.5 \mathrm{mg}$ 
of Benadryl and $100 \mathrm{mg}$ of hydrocortisone intravenously and followed by a small dose of epinephrine 5 ug intravenously three times. Patient regained her baseline blood pressure and heart rate within a few minutes but her coughing and dysphagia were not relieved. Multiple attempts to aspirate from the catheter were unsuccessful. Gradually, the patient developed shortness of breath and chest discomfort. Her oxygen saturations decreased from 100 to 80 and did not respond to oxygen administration through face mask. Auscultation of the lungs revealed generalized crackles especially on right side. Testing of sensation to cold revealed sensory block up to T4. No significant motor weakness was found. Neither ptosis nor miosis was noted. The patient remained in pain free labor for more than three hours and delivered a healthy baby. A follow up Chest CT Scan revealed patchy bilateral infiltrates predominantly in the right lower lobe that was suggestive of aspiration. The patient was discharged home with no sequelae after 3 days medical treatment.

\subsection{Case 2}

A 24-year-old multigravida female with past medical history of depression, recurrent urinary tract infections, and preeclampsia, presented at 37 weeks gestation with rupture of membranes. Patient was allergic to codeine.

On arrival to labor and delivery floor, the patient's blood pressure was 179/95 $\mathrm{mmHg}$ and heart rate 110 beats/min. She received magnesium sulfate intravenously for high blood pressure. The epidural procedure was performed by an experienced physician with the patient in sitting position. The epidural space was identified using loss of resistance to air technique with an 18 gauge Touhy needle. The bevel of the needle was oriented cephalad. An epidural catheter (multiport, opened tip) was placed through the needle with minimal resistance. After a negative aspiration of blood and cerebrospinal fluid from epidural catheter, a test dose of $3 \mathrm{ml}$ of $1.5 \% \mathrm{Li}$ docaine and 1:200,000 epinephrine was injected. This was negative for intravascular and intrathecal placement. A loading dose of $5 \mathrm{ml}$ of $0.25 \%$ of Bupivicaine was administered by slow incremental injection with the patient in supine position. Approximately fifteen minutes after the loading dose was given, the patient began to complain of posterior aspect of neck pain with tinnitis. Systolic blood pressure decreased to $89 \mathrm{mmHg}$ from 150 - $170 \mathrm{mmHg}$ and heart rate decreased to 82 from 102 beat/min, followed by unresponsiveness to verbal stimuli for approximately three minutes. Magnesium sulfate was stopped, aggressive hydration, oxygen via mask and phenylephrine $0.2 \mathrm{mg}$ were administered immediately. Patient regained consciousness in 1 - 2 minutes and her blood pressure came back to normal within a few minutes of treatment. Although the patient complained of difficulty breathing, her oxygen saturation remained $100 \%$. Testing revealed sensory block up to T10. No significant motor weakness was found. The epidural catheters were removed. The patient remained in pain-free labor for more than two hours before delivering a healthy baby.

\section{Discussion}

The subdural space is a potential space that extends throughout the cranium and ends in the lower body of the second sacral vertebra. Most authors describe the subdural space as widest in proportion at the cervical area and narrowest in the lumbar area [1]. The authors postulate that the sparing of sympathetic and motor functions is related to the unique anatomy of this space. The space laterally extends over the exiting dorsal roots. The arachnoid mater and dura mater are fixed at different points over the dorsal root ganglia and this extends the potential space. This is in contrast to the ventral root where the meninges are attached making the potential space there much smaller. This causes the subdural injections to pool in the posterior segment causing the relative sparing of the anterior nerve roots that carry sympathetic and motor fibers $[5,7,8]$.

The presentation of the subdural block depends on the extent of spread of the local anesthetic. The onset of the block is usually slow and the block lasts for several hours. The sensory block achieved usually appears disproportionate to the volume of drug injected and the sympathetic and motor functions are usually spared or only minimally affected. The profound hypotension and apnea seen with subarachnoid block is usual not seen $[9,10]$.

Furthermore, multiple predisposing factors have been implicated in a subdural block including technical difficulty, excessive manipulation of the needle leading to dural injury, and previous back surgery which distorts anatomical planes [2]. However, some recent retrospecttive studies indicated that it may occur independently of the level of experience of practitioners [11].

In our two cases, after a negative CSF aspiration during testing of the catheter, both patients developed a dense sensory block in about ten to fifteen minutes. The sensory block lasted for two to three hours and was followed by full recovery. There was no significant motor block. The cardiovascular instability was mild.

Several studies discuss the diagnosis of subdural block $[2,4,10]$. There are two major and three minor criteria for the diagnosis that are described by Lubenow et al.'s diagnostic paradigm. A negative aspiration and unexpected extensive sensory block are considered by the authors to be major criteria. Also considered as minor criteria aresensory or motor nerve blockade with delayed onset of greater than 10 minutes and a variable motor blockade, 
and sympatholysis out of proportion to the dosage of local anesthetic administered. If both of the major criteria and one of the minor criterions are met, then a subdural blockade should be considered [4]. In addition, a four step algorithm has been proposed by Hoftman and Ferrante to detect subdural blockade. The initial step of this algorithm requires the provider to assess whether the block is thought to be epidural or subarachnoid based on tactile feel upon insertion and also the absence or presence of CSF. The next step involves assessing detmatomal spread. It is graded as excessive, restricted, or neither. The last step involves looking at minor criteria including: onset greater than 20 minutes, cardiovascular stability, sparing of motor fibers, patchy spread, asymmetrical distribution, respiratory failure and cranial nerve involvement [2].

Based on these criteria our index of suspicion for subdural placement in our two cases was high. Both of our cases meet the two major criteria of Lubenow's diagnostic paradigm including a negative aspiration test and unexpected extensive sensory block and several of his minor criteria including a delayed onset by 10 minutes; more of a sensory block; no motor nerve block; sympatholysis out of proportion to the administered dose of local anesthetic. Our cases also fit the diagnostic algorithm proposed by Hoftman and Ferrante which included absence of CSF during testing of the catheter; dermatomal spread assessed as excessive; slow onset; mild cardiovascular instability; motor sparing and cranial involvement.

Cranial nerve block during accidental subdural block is rare but has been reported. In our first patient, the presentation included non productive cough and difficulty in swallowing. This was followed by decreased oxygen saturation. Her chest $\mathrm{X}$-ray was suggestive of aspiration pneumonia. This might have been caused by cephalic spread of local anesthetic to involve the superior and recurrent laryngeal nerves. There were no signs of Horner's syndrome and trigeminal nerve palsy which usually appeared more often than other cranial nerve involvement $[12,13]$. In our second patient we postulate that the excruciating neck pain may have been caused by a sudden CSF pressure change due to the cephalic spread of local anesthetics. The loss of consciousness may have been related to transient hypotension.

Although the other possible differential diagnosis of total spinal block, massive epidural and anaphylaxiswere our concerns, the symptoms were displayed more clearly in favor of the diagnosis of subdural block.

No guideline for the management of a potential subdural catheter has been established. Literature indicates that there is no therapeutic benefit from radiological confirmation of the position of epidural catheter is. In addition, radiological evaluation might contribute to further complications [14]. Also, computed tomography and magnetic resonance imaging scans were not always possible for confirmation of catheter position. Based on this literature, we removed the epidural catheter without radiological evaluation of catheter position.

The awareness of potential presentation of subdural block is key to the timely management of this complication. Even though both patients were hemodynamically stable with fluid resuscitation and small doses of vasopressors, it was warranted to continue close monitoring and supportive therapy for several hours.

\section{Conclusion}

Although subdural catheter placement is a relatively rare occurrence, it is imperative for anesthesiologists to recognize the presentation and treat accordingly.

\section{REFERENCES}

[1] H. Gray, “Anatomy of the Human Body,” 30th Edition, Lea \& Febiger, Philadelphia, 1985, p. 1125.

[2] T. Lubenow, E. Keh-Wong, K. Kristof, O. Ivankovich and A. D. Ivankovich, "Inadvertent Subdural Injection: A Complication of an Epidural Block,” Anesthesia \& Analgesia, Vol. 67, No. 2, 1988, pp. 175-179.

[3] W. P. Milants, P. M. Parizel, J. de Moor, I. G. Tobback and A. M. De Schepper, "Epidural and Subdural Contrast in Myelography and CT Myelography,” European Journal of Radiology, Vol. 16, No. 2, 1993, pp. 147-150. doi:10.1016/0720-048X(93)90013-D

[4] N. N. Hoftman and F. M. Ferrante, "Diagnosis of Unintentional Subdural Anesthesia/Analgesia: Analyzing Radiographically Proven Cases to Define the Clinical Entity and to Develop a Diagnostic Algorithm," Regional Anesthesia \& Pain Medicine, Vol. 34, No. 1, 2009, pp. 12-16. doi:10.1097/AAP.0b013e31819339cf

[5] R. M. G. Pearson, "A Rare Complication of Extradural Analgesia,” Anaesthesia, Vol. 39, No. 5, 1984, pp. 460463. doi:10.1111/j.1365-2044.1984.tb07315.x

[6] B. C. Tsui, S. Gupta, D. Emery and B. Finucane, "Detection of Subdural Placement of Epidural Catheter Using Nerve Stimulation," Canadian Journal of Anesthesia, Vol. 47, No. 5, 2000, pp. 471-473. doi:10.1007/BF03018980

[7] R. Shapiro, "Myelography,” 3rd Edition, Year Book Medical Publishers, Chicago, 1975, p. 124.

[8] G. J. Romanes, “Cunningham's Manual of Practical Anatomy,” 14th Edition, Oxford University Press, Oxford, 1986.

[9] C. Collier, "Total Spinal or Massive Subdural Block," Anaesthesia and Intensive Care, Vol. 10, No. 1, 1982, pp. 92-93.

[10] E. Abouleish and M. Goldstein, "Migration of an Extradural Catheter into the Subdural Space: A Case Report," British Journal of Anesthesia, Vol. 58, No. 10, 1986, pp. 1194-1197. doi:10.1093/bja/58.10.1194

[11] D. Agarwal, M. Mohta, A. Tyagi and A. K. Sethi, "Sub- 
dural Block and the Anaesthetist," Anaesthesia and Intensive Care, Vol. 38, No. 1, 2010, pp. 20-26.

[12] J. Rodríguez, M. Bárcena, M. Taboada-Muñiz and J. Alvarez, "Horner Syndrome after Unintended Subdural Block. A Report of 2 Cases,” Journal of Clinical Anesthesia, Vol. 17, No. 6, 2005, pp. 473-477. doi:10.1016/j.jclinane.2004.09.010

[13] F. De la Gala, A. Reyes, M. Avellanal, P. Baticón and L.
M. González-Zarco, “Trigeminal Nerve Palsy and Horner's Syndrome Following Epidural Analgesia for Labor: A Subdural Block?” International Journal of Obstetric Anesthesia, Vol. 16, No. 2, 2007, pp. 180-182. doi:10.1016/j.ijoa.2006.07.007

[14] G. T. Bell and J. C. Taylor, "Subdural Block-Further Points," Anaesthesia, Vol. 49, No. 9, 1994, pp. 794-795. doi:10.1111/j.1365-2044.1994.tb04455.x 